\title{
Combined Reversed Phase HPLC, Mass Spectrometry, and NMR Spectroscopy for a Fast Separation and Efficient Identification of Phosphatidylcholines
}

\author{
Jan Willmann, ${ }^{1}$ Herbert Thiele, ${ }^{2}$ and Dieter Leibfritz ${ }^{1}$ \\ ${ }^{1}$ Institute of Organic Chemistry, University of Bremen, NW2C, 28359 Bremen, Germany \\ ${ }^{2}$ Bruker Daltonik GmbH, Fahrenheitstr. 4, 28359 Bremen, Germany \\ Correspondence should be addressed to Dieter Leibfritz, dieter.leibfritz@uni-bremen.de \\ Received 14 May 2010; Accepted 21 July 2010 \\ Academic Editor: Olav Kvalheim
}

Copyright (C) 2011 Jan Willmann et al. This is an open access article distributed under the Creative Commons Attribution License, which permits unrestricted use, distribution, and reproduction in any medium, provided the original work is properly cited.

In respect of the manifold involvement of lipids in biochemical processes, the analysis of intact and underivatised lipids of body fluids as well as cell and tissue extracts is still a challenging task, if detailed molecular information is required. Therefore, the advantage of combined use of high-pressure liquid chromatography (HPLC), mass spectrometry (MS), and nuclear magnetic resonance (NMR) spectroscopy will be shown analyzing three different types of extracts of the ubiquitous membrane component phosphatidylcholine. At first, different reversed phase modifications were tested on phosphatidylcholines (PC) with the same effective carbon number (ECN) for their applicability in lipid analysis. The results were taken to improve the separation of three natural PC extract types and a new reversed phase (RP)-HPLC method was developed. The individual species were characterized by one- and two-dimensional NMR and positive or negative ion mode quadrupole time of flight (q-TOF)-MS as well as MS/MS techniques. Furthermore, ion suppression effects during electrospray ionisation (ESI), difficulties, limits, and advantages of the individual analytical techniques are addressed.

\section{Introduction}

The analysis of native and underivatized lipids within body fluids as well as cell and tissue extracts is still a challenging task, in particular, if the molecular structure of individual components needs to be identified in decently short time. The lipid composition consists of different main classes such as fatty acids, neutral lipids, and lipids with positively or negatively charged head groups with manifold subclasses of structural diversity. Variations within the lipid composition were attributed to different pathologies such as neoplastic and neurodegenerative diseases, diabetes mellitus, and many others. Furthermore, some lipid classes are involved in cell death (apoptosis, necrosis), cellular signaling and are precursors for lysophospholipids (i.e., lysophosphatidylcholine), diacylglycerols, and phosphatic and arachidonic acid [1-25].

1,2-Diacyl-sn-glycero-3-phosphatidylcholine (PC) represents a major constituent of cell membranes. It consists of the polar head group phosphorylcholine attached to the sn-3 position of glycerol and differing saturated and unsaturated fatty acids esterified to the sn- 1 and sn-2 position, whereby fatty acids in position sn-1 are preferentially saturated as a rule. Numerous studies dealt with PCs in the past because of their utmost biochemical and clinical importance and many different analytical techniques have been proposed to get an insight into metabolic turnover or to characterize pathophysiological deviations of the native lipid composition. Most of these techniques suffer from various drawbacks as being time-consuming, insensitive, destructive, or not related to individual substructures. Gas chromatography (GC) [2628], thin layer chromatography (TLC) [29-31], and highperformance liquid chromatography (HPLC) [32-37] are commonly used for lipid analysis. GC-based techniques are quantitative but require time-consuming sample preparation techniques. GC is often used in combination with TLC for the lipid class separation. The spots on a TLC plate are 
scratched out and their fatty acid residues are analyzed upon derivatization into a volatile substrate and recorded by GC. However, the precise molecular structure of an individual lipid is lost because of the preceding hydrolysis of the lipids. Enzymatic cleavage of the ester bond using phospholipases allows a successive hydrolysis of the sn- 2 and sn- 1 fatty acid, but it is rather time-consuming due to intense laboratory work and already minor contamination of the enzyme leads to false results. HPLC offers the separation of lipid classes using the normal phase mode (NP) and additionally the separation according to the different fatty acid residues of an individual lipid in the reversed phase mode (RP). In this case, a successful separation depends distinctly on the appropriate selection of the stationary phase. Alternatively, MS-based techniques are widely used, as they are fast, sensitive and require only minor sample preparation [38]. The use of high resolution MS systems give access to the molecular formula. In addition, characteristic fragmentations identify the lipid class and molecular structure. When coupled with a HPLC-system their selectivity is much higher and benefits from both techniques. NMR spectroscopy is capable to measure intact biomaterials nondestructively without any preceding derivatization. Especially ${ }^{31} \mathrm{P}-\mathrm{NMR}$ is well-suited to quantify phospholipid class analysis and needs only less sample preparation [39-44]. Again only minor information is obtained with respect to the fatty acid residues. ${ }^{1} \mathrm{H}$ NMR measurements are also widely used, as they contain more information about the fatty acids in general, but the connection to the glycerol backbone is missing due to massive signal overlap. $2 \mathrm{D}-\mathrm{NMR}$ involving the ${ }^{13} \mathrm{C}$ nucleus provides a lot more resolution and more information about individual species, but the low NMR sensitivity of the ${ }^{13} \mathrm{C}$ isotope prevents a fast and wide application of this technique in a routine analysis [44-48].

This paper presents an efficient RP-HPLC setup to separate phosphatidylcholines, which ultimately will be extendable to separate other polar phospholipids. Subsequently the HPLC tool is combined with the highly informative molecular assignment potentials of MS and NMR [49]. Five different types of silica-based reversed phase modifications were tested with respect to their capability to separate lipids containing fatty acids with an equivalent carbon number (ECN), which is the number of carbon atoms within a fatty acid chain minus twice the number of double bonds. The extension of a lipid by a $\mathrm{C}=\mathrm{C}$ double bond will not change the hydrophobicity. The performance of all columns was tested on a mixture of five PCs with the same ECN whereas two of them are even constitutional isomers concerning the 1,2-positions of glycerol, which hampers the separation even more.

Then, the HPLC column with best performance was used to achieve an efficient baseline separation of three native PC extracts (soy bean, bovine brain, and egg yolk). Furthermore, the MS fragmentation behavior in the positive and negative ion mode is investigated for individual PCs to identify characteristic fragmentation patterns for this lipid class and its fatty acid residues. $1 \mathrm{D}$ and $2 \mathrm{D}$ high-resolution NMR spectra were also acquired to confirm the molecular structure.

\section{Material and Methods}

2.1. Chemicals. Methanol-d4 and deuterated chloroform, methanol (LC-MS grade), all fatty acids, the test mixture compounds dipalmitoyl-phosphatidylcholine (DPPC), palmitoyl-oleoyl-phosphatidylcholine (POPC), oleoyl-palmitoyl-phosphatidylcholine (OPPC), dioleoyl-phosphatidylcholine (DOPC), stearoyl-linoleoyl-phosphatidylcholine (SLPC), and also the soy bean, bovine brain, and egg yolk extracts were purchased from Sigma-Aldrich Chemie GmbH (Taufkirchen, Germany). The double distilled water was taken from the in-house system.

2.2. High-Performance Liquid Chromatography. A HP 1100 series HPLC system (Agilent Technologies, Waldbronn, Germany) was used. The injection volume was $3 \mu \mathrm{L}$ of the standard prepared in methanol. Five columns with different stationary phases were tested with respect to their separation performance for lipid analysis:

(1) type A silica-based endcapped $\mathrm{C}_{18}$ (Nucleosil 100-5 C18, $250 \times 3 \mathrm{~mm}$ ),

(2) type A silica-based phenyl (Nucleosil 100-5 $\mathrm{C}_{6} \mathrm{H}_{5}$, $250 \times 4 \mathrm{~mm})$,

(3) type $\mathrm{B}$ silica-based high density $\mathrm{C}_{18}$ (Nucleodur C18 Gravity, $5 \mu \mathrm{m}, 250 \times 3 \mathrm{~mm})$,

(4) type B silica-based polymer/cross linked $\mathrm{C}_{18}$ (Nucleodur C18 Isis, $5 \mu \mathrm{m}, 250 \times 3 \mathrm{~mm}$ ),

(5) type B silica-based mixed mode phenyl/C ${ }_{18}$ (Nucleodur Sphinx RP, $5 \mu \mathrm{m}, 250 \times 3 \mathrm{~mm})$.

All HPLC columns and materials were a kind gift of Macherey-Nagel (Düren, Germany).

The $3 \mathrm{~mm}$ columns were operated at flow rate of $0.6 \mathrm{~mL} / \mathrm{min}$ and the $4 \mathrm{~mm}$ column at $1 \mathrm{~mL} / \mathrm{min}$. The mobile phase was optimized by adapting the methanol content in different runs between $90 \%$ and $100 \%$ for the alkyl phases and between $80 \%$ and $100 \%$ for the phenyl phase with respect to the hydrophobic interaction of the analytes with the RP packing.

An $8 \mathrm{~mm}$ Nucleodur Sphinx RP was operated under isocratic conditions at $4.1 \mathrm{~mL} / \mathrm{min}$ flow with a mobile phase consisting of methanol and water $(90: 10)$ for the semi preparative approach. To collect the individual species for NMR measurements a Gilson 215 liquid handler (Gilson International B.V., Bad Camberg, Germany) was used. The column temperature was kept at $40^{\circ} \mathrm{C}$ in all runs.

2.3. Mass Spectrometry. An esquire LC iontrap system (Bruker Daltonik GmbH, Bremen, Germany) was used for mass spectrometric detection for positive and negative ion mode mass and MS/MS spectra of each PC compound were recorded. The capillary voltage was set to $-3800 \mathrm{~V}$ and the end plate offset to $500 \mathrm{~V}$ in positive ion mode. For the HPLC the nebulizer gas was set to 40 psi, dry gas and dry heat were set to $10 \mathrm{~L} / \mathrm{min}$ and $300^{\circ} \mathrm{C}$, respectively. In case of direct infusion via a syringe pump, the dry and nebulizer gases were reduced to $5 \mathrm{~L} / \mathrm{min}$ and 5 psi, respectively. The collision 
energy for MS/MS experiments was optimized with respect to the precursor ion stability.

A micrOTOF-Q-equipped with the Apollo ESI ion source (Bruker Daltonik GmbH, Bremen, Germany) was used for precision mass detection. The capillary voltage was set to $4500 \mathrm{~V}$ and the end plate offset to $-500 \mathrm{~V}$ in negative ion mode. The nebulizer gas was set to 0.4 bar, dry gas and dry heat were set to $4 \mathrm{~L} / \mathrm{min}$ and $200^{\circ} \mathrm{C}$, respectively. For MS/MS experiments the collision energy of the quadrupole was $-42 \mathrm{eV} / \mathrm{z}$. The molecular formula was generated by matching high mass accuracy and isotopic pattern (SigmaFit, Bruker Daltonik GmbH, Bremen, Germany).

2.4. Nuclear Magnetic Resonance. All samples were stored at $-80^{\circ} \mathrm{C}$ before the measurements. In case of dissolved samples, the solvents were evaporated by a gentle stream of nitrogen and redissolved in $\mathrm{CDCl}_{3} / \mathrm{CD}_{3} \mathrm{OD}(2: 1)$. $1 \mathrm{D}$ $\left({ }^{1} \mathrm{H},{ }^{13} \mathrm{C}\right)$ and high-resolution 2D (HSQC, HSQC-TOCSY, HMBC) NMR spectra with a digital resolution of $1 \mathrm{k}$ data points in $\mathrm{F} 1$ and $4 \mathrm{k}$ data point in $\mathrm{F} 2$ dimension of each PC species were acquired on a Bruker DRX $600 \mathrm{MHz}$ NMR spectrometer equipped with $5 \mathrm{~mm}$ TXI probe (Bruker BioSpin GmbH, Rheinstetten/Karlsruhe, Germany).

\section{Results}

3.1. High-Performance Liquid Chromatography. A comparison of five different reversed phase columns revealed the following behavior: The separation of the test mixture on type A silica-based materials showed only poor results for all PC compounds. Although, it seems that the Nucleosil material separates all peaks very well (see Table 1), the extreme peak broadening and a distinct tailing spoils the pretended peak separation. In contrast, the type B silica based materials separated DPPC, DOPC, SLPC, and POPC or OPPC very well. However, the two lipid isomers POPC and OPPC were only well separated (Table 1) on the polymer cross link RP packing (ISIS). With all RP materials it was possible to separate lipids containing two monounsaturated fatty acids from lipids with one or two saturated or one polyunsaturated fatty acid. The shortest separation times with sharp chromatographic peaks were achieved by the mixed mode stationary phase (Sphinx). Therefore, this stationary phase was selected to separate the individual compounds within the lipid extracts of natural sources.

3.1.1. Separation of the Phosphatidylcholine Extracts. The mixed mode stationary phase (Sphinx) and mobile phase of $90 \%$ methanol and $10 \%$ water allows the baseline separation of all species within the three different extracts (i.e., extraction residues). The separation of the PC species is not influenced by the type or contaminations of the extract. The results are listed in Table 2.

Altogether, twelve PC components and one plasmalogene (bovine brain extract) were identified. All species contained a saturated fatty acid in position sn-1 (i.e., myristic (1 species), palmitic (7 species) or stearic acid (4 species). Palmitic or stearic acid were esterified to the sn-2 position within three

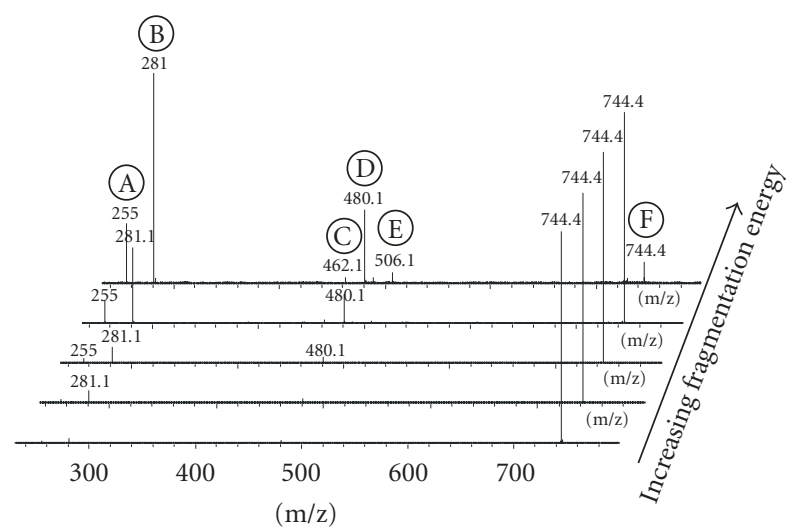

FIGURE 1: Variation of the fragmentation energy of negative ion mode ESI-MS² of POPC (744.4).

compounds. The other nine compounds contained a mono(four times) or polyunsaturated fatty acid (five times) in position sn-2. The plasmalogen consists of octadecanol (sn1) and oleic acid (sn-2). Most species were identified in bovine brain extract $(11+1)$ and the fewest in the egg yolk extract (8).

3.1.2. Preparation of the Individual Compounds for NMR Measurement. The same mobile phase $(90 \%$ methanol and $10 \%$ water) and column type (MN Sphinx) were used for the semipreparative HPLC runs as for the analytical measurements. However, the HPLC column dimension was upscaled ( $8 \mathrm{~mm}$ instead of $3 \mathrm{~mm}$ ). The flow was splitted after the column and a small amount was used for peak detection and identification into the micro-TOF-Q. The residual eluent was collected for NMR measurements.

3.2. Mass Spectrometry. The ionization efficiencies of all equimolar concentrated saturated fatty acids were recorded relative to the internal standard undecanoic acid in different measurements using the negative ESI ion mode. The ion counts of undecanoic acid were set to 100 percent in all cases and the ion counts of all other fatty acids were recalculated with respect to this value (Table 3 ). All fatty acids with a shorter chain length as the internal standard show lower ionization efficiencies and all fatty acids with 12 or more carbon atoms show higher ionization efficiencies. The ionization efficiency of the fatty acids increases not linearly.

3.2.1. Fragmentation. The positive ion mode MS spectra showed better signal to noise ratios than in the negative ion mode. In positive ion mode, the base peak results from the adsorption of a sodium ion. In negative ion mode, the base peak results from the demethylation of the parent ion during the transfer into the ion trap mass spectrometer. $[\mathrm{M}+\mathrm{A}]^{-}$, whereby $\mathrm{A}$ is chloride or formate, was observed to a small extent only. In positive ion mode, MS/MS the polar headgroup of phosphorylcholine was cleaved off. In negative ion mode, MS/MS spectra the fatty acids of each 
TABLE 1: Retention times of different PC-components with the same ECN on different reversed phase columns.

\begin{tabular}{lccccc}
\hline Lipid & & \multicolumn{2}{c}{ Column type } \\
& Nucleosil 100-5 $\mathrm{C}_{6} \mathrm{H}_{5}$ & Nucleosil 100-5 C18 & Nucleodur C18 Gravity & Nucleodur Sphinx RP & Nucleodur C18 Isis \\
\hline DPPC & $1.46(5.76)^{*}$ & 56.82 & 78.00 & 14.31 & 49.19 \\
OPPC & $1.52(6.49)^{*}$ & 59.27 & 86.00 & 16.26 & 51.81 \\
POPC & $1.52(6.48)^{*}$ & 57.36 & 86.00 & 16.26 & 52.19 \\
DOPC & $1.64(7.30)^{*}$ & 57.18 & 98.67 & 19.11 & 56.40 \\
SLPC & $1.64(7.58)^{*}$ & 69.95 & 107.00 & 19.66 & 60.81 \\
\hline
\end{tabular}

* mobile phase methanol/water $(80: 20)$.

TABLE 2: Identified PC compounds within extracts of bovine brain, egg yolk, and soy bean.

\begin{tabular}{|c|c|c|c|c|c|}
\hline \multicolumn{2}{|c|}{ Fatty acid position } & \multirow{2}{*}{ ECN } & \multirow{2}{*}{ Bovine brain } & \multirow{2}{*}{ Egg yolk } & \multirow{2}{*}{ Soy bean } \\
\hline sn-1 position & sn-2 position & & & & \\
\hline Myristic acid(14:0) & Palmitic acid $(16: 0)$ & 30 & + & - & + \\
\hline Palmitic acid $(16: 0)$ & Palmitoleic acid (16:1) & 30 & + & + & + \\
\hline Palmitic acid $(16: 0)$ & Linoleic acid $(18: 2)$ & 30 & + & + & + \\
\hline Palmitic acid $(16: 0)$ & Linolenic acid $(18: 3)$ & 28 & - & + & - \\
\hline Palmitic acid $(16: 0)$ & Arachidonic acid $(20: 4)$ & 28 & + & + & + \\
\hline Palmitic acid $(16: 0)$ & Palmitic acid $(16: 0)$ & 32 & + & + & + \\
\hline Palmitic acid $(16: 0)$ & Oleic acid $(18: 1)$ & 32 & + & + & + \\
\hline Stearic acid $(18: 0)$ & Linoleic acid $(18: 2)$ & 32 & + & + & + \\
\hline Stearic acid $(18: 0)$ & Arachidonic acid $(20: 4)$ & 30 & + & - & + \\
\hline C18: 0 Plasmalogen & Oleic acid $(18: 1)$ & 34 & + & - & - \\
\hline Palmitic acid $(16: 0)$ & Stearic acid $(18: 0)$ & 34 & + & - & - \\
\hline Stearic acid $(18: 0)$ & Oleic acid $(18: 1)$ & 34 & + & + & + \\
\hline Stearic acid $(18: 0)$ & Eicosenoic acid $(20: 1)$ & 36 & + & - & - \\
\hline
\end{tabular}

TABle 3: Electrospray ionization efficiency of different fatty acid compared to undecanoic acid ( $p K_{s}=4,69$ for all acids).

\begin{tabular}{lccc}
\hline Fatty acid & $\begin{array}{c}\text { Number of } \\
\text { carbons }\end{array}$ & $\begin{array}{c}\text { Empirical } \\
\text { formula }\end{array}$ & $\begin{array}{c}\text { Ionisation } \\
\text { efficiency* (\%) }\end{array}$ \\
\hline caprylic acid & 8 & $\mathrm{C}_{8} \mathrm{H}_{16} \mathrm{O}_{2}$ & 52 \\
capric acid & 10 & $\mathrm{C}_{10} \mathrm{H}_{20} \mathrm{O}_{2}$ & 64 \\
undecanoic acid & 11 & $\mathrm{C}_{11} \mathrm{H}_{22} \mathrm{O}_{2}$ & 100 \\
lauric acid & 12 & $\mathrm{C}_{12} \mathrm{H}_{24} \mathrm{O}_{2}$ & 131 \\
myristic acid & 14 & $\mathrm{C}_{14} \mathrm{H}_{28} \mathrm{O}_{2}$ & 194 \\
palmitic acid & 16 & $\mathrm{C}_{16} \mathrm{H}_{32} \mathrm{O}_{2}$ & 285 \\
stearic acid & 18 & $\mathrm{C}_{18} \mathrm{H}_{36} \mathrm{O}_{2}$ & 609 \\
eicosanoic acid & 20 & $\mathrm{C}_{20} \mathrm{H}_{40} \mathrm{O}_{2}$ & 2300 \\
\hline
\end{tabular}

* compared to undecanoic acid.

PC were assigned by detection of their $[\mathrm{M}-\mathrm{H}]^{-}$ion accompanied by a neutral loss of the fatty acid ketene. The sn-2 fatty acid of every PC species shows always the more intense signal compared to the sn-1 fatty acid signal. Figure 1 shows several overlaid MS/MS iontrap spectra of POPC, but with increasing fragmentation energy to show the energy dependence of the different fragment ions. Table 4 lists the individual observed fragments and their corresponding $\mathrm{m} / \mathrm{z}$ values.
3.2.2. Identification of the Phosphatidylcholines in the Different Extracts. Negative ion mode MS and auto-MS/MS q-TOF spectra were recorded during the HPLC runs. The fatty acid residues of each individual PC component was assigned by its relative fragment ion intensities. Furthermore, the molecular formula of each detected ion was generated by matching high mass accuracy and isotopic pattern to confirm the results. The lipid class was confirmed by the molecular formula and reconstructing the precursor ion of the fragment ions. Table 2 shows the indentified species within the three different extracts.

3.3. Nuclear Magnetic Resonance. $1 \mathrm{D}-\left({ }^{1} \mathrm{H}\right.$ and $\left.{ }^{13} \mathrm{C}\right)$ and $2 \mathrm{D}-$ (HSQC, HSQC-TOCSY, and HMBC) NMR spectra of the five reference PCs were recorded for peak assignment. The NMR parameters of all reference compounds are listed in the supplements. Lipids with saturated (DPPC), mono unsaturated fatty acids (MOFA) as in POPC, OPPC, DOPC, or polyunsaturated fatty acids (PUFA) as in SLPC show zero, two or four carbon signals between 120 and $130 \mathrm{ppm}$. The MOFA and PUFA show unambiguously different chemical shifts for the olefinic carbons; however, the three lipids with MOFAs have nearly identical olefinic carbon shifts respectively the distance between the double bond signals depends on the MOFA location (sn-1 or sn-2). Figure 2 
TABLE 4: Assignment of the individual observed fragments of POPC.

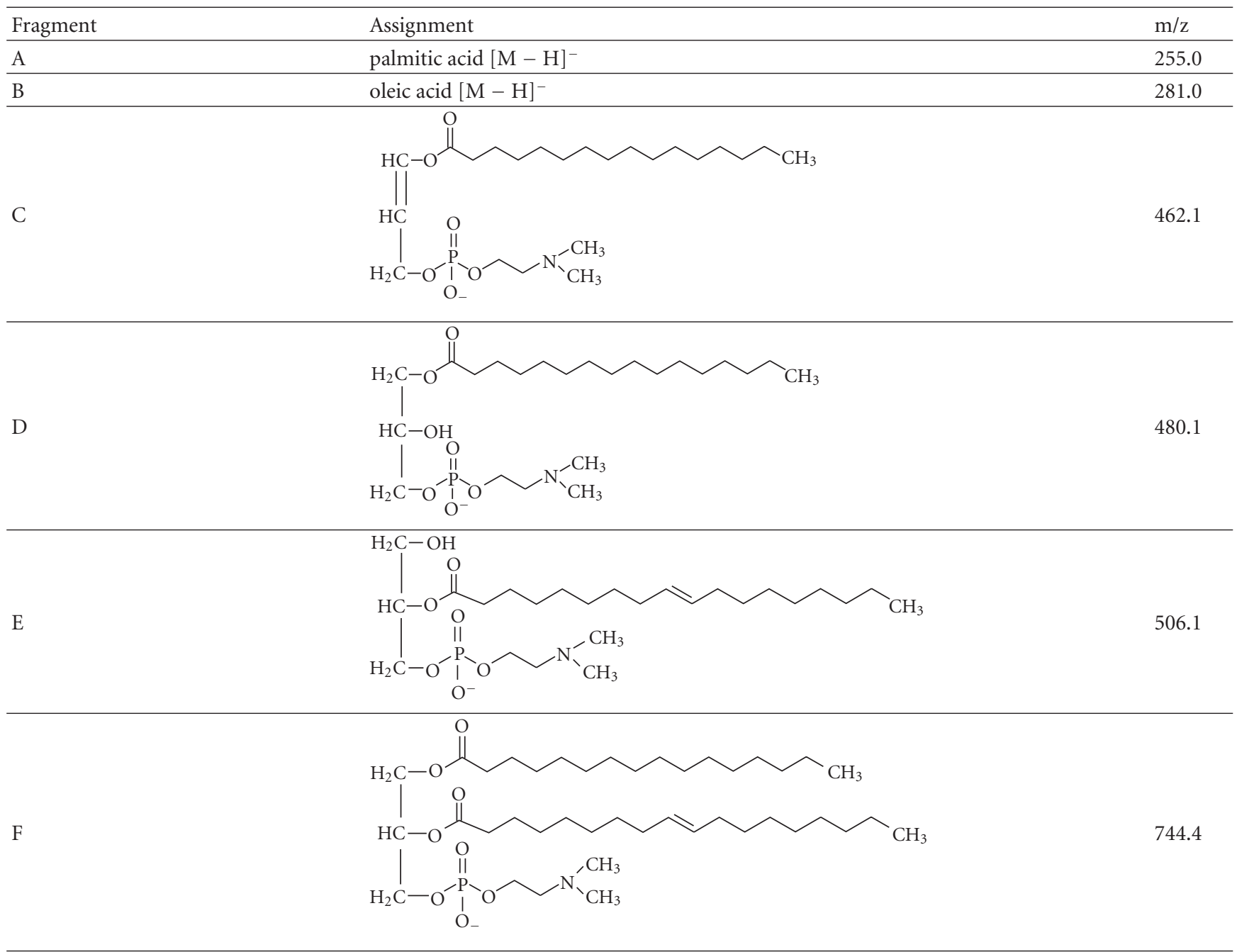

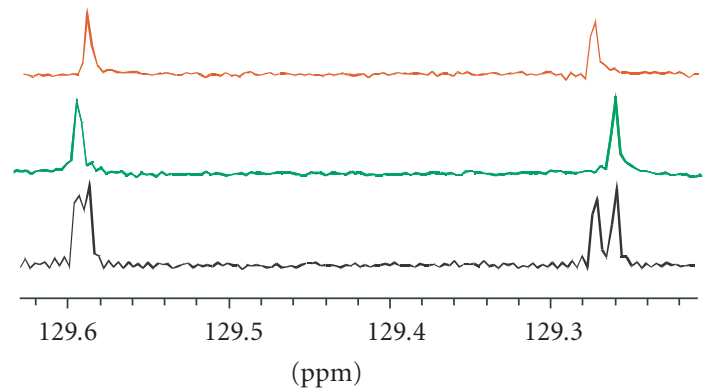

FIGURE 2: Overlaid ${ }^{13} \mathrm{C}$-NMR spectral sections of olefinic carbons of PCs with MOFAs (top OPPC, middle POPC, and bottom DOPC).

shows the overlaid ${ }^{13} \mathrm{C}$ NMR spectra of POPC, OPPC, and DOPC. The chemical shift difference between these two signals is about $50 \mathrm{~Hz}$ for POPC and about $47 \mathrm{~Hz}$ for OPPC. The corresponding ${ }^{1} \mathrm{H}$-NMR spectra show identical signals for the olefinic protons (not shown). Nonetheless, a lipid with two MOFAs can be deduced from the intensity ratio of the olefinic protons to the glycerol protons in the ${ }^{1} \mathrm{H}-\mathrm{NMR}$ spectra.

3.3.1. NMR of the PC Extracts. HPLC-MS structural results were confirmed for all molecular species using $1 \mathrm{D}-\left({ }^{1} \mathrm{H}\right)$ and 2D-(HSQC, HSQC-TOCSY, and HMBC) NMR techniques. The results are shown in Table 2. No branched chain or oxidized fatty acids were observed. The NMR parameter of unsaturated fatty acids have been measured separately (see Supplement) and are identical within the mixtures.

\section{Discussion}

Lipids with equal ECN should have the same hydrophobicity, which is the discriminating factor in reversed phase chromatography. This holds for PCs with the same ECN within a mixture and even more, if they are stereoisomers such as POPC and OPPC. The successful reversed phase separation of hydrophobic and zwitterionic molecules like phosphatidylcholines depends on very well endcapped silica materials, as Coulomb interactions of the choline group 
with the silica gel lead to peak broadening, retention time shifts (data not shown), and peak tailing. Packing materials with hydrophobic van der Waals interactions only (i.e., high density C18 materials) show no separation of isomeric lipids. Additional interactions like steric effects by polymer crosslinked RP packings can overcome this problem. Stationary phases with only aromatic modifications are not hydrophobic enough to achieve a good phospholipid separation. The mixed mode stationary phase contains additionally alkyl ligands and offers therefore more hydrophobic interactions and good silica gel coverage. This column showed the shortest phospholipid retention times for all alkyl stationary phases and a good separation with narrow chromatographic peaks. Saturated lipids have the lowest and polyunsaturated lipids the highest retention times. Comparing lipids of the same ECN with two MOFAs or one PUFA and one saturated fatty acid the retention of the lipid with the PUFA is higher, as their spatial demand is larger and the $\pi-\pi$ interactions with a PUFA residue is not affected by the other fatty acid.

4.1. Separation of the Phosphatidylcholine Extracts. By means of the HPLC separation it was possible to get semiquantitative information of the individual compounds in the extracts. Referring to the results of the separation of the lipid standard mixture it was possible to separate all species within the extracts. The elution order of lipids with the same ECN is in the same line as for the reference mixture (lipids with saturated fatty acids, one MOFA, two MOFAs, one PUFA). However, in case of PUFAs with four double bonds (i.e., arachidonic acid; $20: 4$ ) the $\pi-\pi$ interactions become so strong that this compound elutes as last compound in the next higher ECN group (see Table 2). Nonetheless, they are unambiguously identified by means of their MS fragmentation or NMR spectra.

\subsection{Preparation of the Individual Compounds for NMR} Measurement. The upscaling with the same type of RP packing of the newest generation is no problem, although a peak broadening can occur because of the higher sample load.

4.3. Ion Suppression Effects. The ionization efficiency of the different fatty acids during ESI increases nonlinearly. The results can be correlated with the molecules hydrophobicity, which shows the same progression. The hydrophobicity is obtained from the octanol-water partitioning coefficient $\left(\log \mathrm{p}_{\mathrm{ow}}\right)$ (data not shown). There are no differences between the $\mathrm{p} K_{\mathrm{a}}$ values of the individual fatty acids (Table 3 ), so that the ionization efficiency of the fatty acids depends only on the molecules hydrophobicity, which increases by the number of carbon atoms.

As POPC and OPPC or SLPC and DOPC, respectively, have the same molecular weight/precision mass, they cannot be assigned based on their mol peak only. However, they are distinguished by MS/MS spectra, as the sn-2 fatty acid is always cleaved off at lower fragmentation energies due to sterical effects. In addition, the intensity ratio of the sn-2 fatty acid anion to the sn-1 fatty acid anion is constant for a particular fragmentation energy. A semiquantitative or even quantitative analysis of stereoisomeric PCs within a mixture (f.e., POPC and OPPC) still is difficult, because the anion signal of an individual fatty acid will be the same regardless the position on the glycerol backbone and no other distinguishing signal is observed for one of the isomers. However, using the intensity ratio of the sn- 2 fatty acid anion to the sn- 1 fatty acid anion for the pure reference compound one can calculate the approximate POPC to OPPC ratio.

The MS detection limits also benefits from the isocratic HPLC method with high-organic solvent concentration in the mobile phase. In case of gradient HPLC, methods the ionization efficiency varies with the varying organic percentage of the mobile phase. Furthermore, the risk of ion suppression during the ionization process is minimized by the sample introduction after HPLC separation.

The identification of the individual species in the different extracts was achieved by MS/MS and comparing the individual fragment intensities as described earlier. The use of high-resolution spectra acquired by the micro-TOF-Q allows the generation of the compound's molecular formula by matching high mass accuracy and isotopic pattern. Abnormalities like oxidation of the double bond, and so forth. were not observed.

It may be noted, that not only diacyl PLs can be identified within a mixture, but also alkyl/alkenyl, acyl PLs because of the different fragmentation pattern of the fatty acid residue compared to an ether link.

The HPLC and MS results were confirmed by NMR spectroscopy, especially the configuration and location of double bonds in the fatty acid residues. Only NMR having the highest qualitative and quantitative structure elucidation potential allows a complete structure elucidation. ${ }^{1} \mathrm{H}-,{ }^{13} \mathrm{C}$-, or ${ }^{31} \mathrm{P}$-NMR spectra are capable to identify the various lipid classes. Beyond this, the degree of unsaturation is obtained from the proton signal intensity ratio of the double bond signal versus the choline group signal. MOFAs and PUFAs are differentiated by the number of carbon signals within the double bond region. The location (sn-1 or sn-2) of the MOFA follows from the ${ }^{13} \mathrm{C}$-NMR spectra or from ESI-MS. The risk of peak overlapping in the NMR spectra was avoided by recording 2D-NMR spectra by preceding separation of individual phosphatidylcholines by HPLC.

\section{Conclusion}

The separation of lipids with equivalent chain lengths in complex mixtures can be improved using RP-HPLC packings of the newer generation containing additional discriminators. Already simple PC mixture cannot be assigned by a single analytical technique, while the combination of HPLC separation power, MS sensitivity with accurate mass measurement of molecular and fragment ions and NMR structure elucidation power will meet most suitably the challenge. They overcome the limits of any single technique and also proof the potential of their combination ultimately to analyze native (lipid) mixtures. The molecular structure of a novel compound may not be evaluated by NMR alone, 
if the native concentration is very low. Conversely, MS data may give molecular weight, fragmentation and molecular formulae that may be insufficient to assign ambiguously the molecular structure of an unknown compound. However, online NMR and MS detections in parallel provide complementary data and minimize ambiguities between LC-MS and LC-NMR systems very efficiently.

Combination of these data allows the identification of a lipid class, reconstruction of the lipid structure, and both the location of an individual fatty acid to the sn- 1 or sn-2 position in the glycerol moiety or the location of double bond within the fatty acid chains.

\section{References}

[1] F. Hullin, M.-J. Bossant, and N. Salem Jr., "Aminophospholipid molecular species asymmetry in the human erythrocyte plasma membrane," Biochimica et Biophysica Acta, vol. 1061, no. 1, pp. 15-25, 1991.

[2] T. Matsura, A. Togawa, M. Kai et al., "The presence of oxidized phosphatidylserine on Fas-mediated apoptotic cell surface," Biochimica et Biophysica Acta, vol. 1736, no. 3, pp. 181-188, 2005.

[3] T. R. Pettitt, S. K. Dove, A. Lubben, S. D. J. Calaminus, and M. J. O. Wakelam, "Analysis of intact phosphoinositides in biological samples," Journal of Lipid Research, vol. 47, no. 7, pp. 1588-1596, 2006.

[4] H.-P. Ma, C.-F. Chou, S.-P. Wei, and D. C. Eaton, "Regulation of the epithelial sodium channel by phosphatidylinositides: experiments, implications, and speculations," Pflügers Archiv European Journal of Physiology, vol. 455, no. 1, pp. 169-180, 2007.

[5] M. J. Berridge, "Inositol trisphosphate and calcium signalling," Nature, vol. 361, no. 6410, pp. 315-325, 1993.

[6] O. Kafrawy, M. Zerouga, W. Stillwell, and L. J. Jenski, "Docosahexaenoic acid in phosphatidylcholine mediates cytotoxicity more effectively than other $\omega-3$ and $\omega-6$ fatty acids," Cancer Letters, vol. 132, no. 1-2, pp. 23-29, 1998.

[7] I. Kan, E. Melamed, D. Offen, and P. Green, "Docosahexaenoic acid and arachidonic acid are fundamental supplements for the induction of neuronal differentiation," Journal of Lipid Research, vol. 48, no. 3, pp. 513-517, 2007.

[8] T. Kolter and K. Sandhoff, "Sphingolipide-ihre stoffwechselwege und die pathobiochemie neurodegenerativer erkrankungen," Angewandte Chemie, vol. 111, pp. 1632-1670, 1999.

[9] R. N. Kolesnick, "Sphingomyelin and derivatives as cellular signals," Progress in Lipid Research, vol. 30, no. 1, pp. 1-38, 1991.

[10] M. Garnier, E. J. Dufourc, and B. Larijani, "Characterisation of lipids in cell signalling and membrane dynamics by nuclear magnetic resonance spectroscopy and mass spectrometry," Signal Transduction, vol. 6, no. 2, pp. 133-143, 2006.

[11] J. H. Exton, "Phosphatidylcholine breakdown and signal transduction," Biochimica et Biophysica Acta, vol. 1212, no. 1, pp. 26-42, 1994.

[12] C. D. Stubbs and A. D. Smith, "The modification of mammalian membrane polyunsaturated fatty acid composition in relation to membrane fluidity and function," Biochimica et Biophysica Acta, vol. 779, no. 1, pp. 89-137, 1984.

[13] J. Adachi, N. Yoshioka, M. Sato, K. Nakagawa, Y. Yamamoto, and Y. Ueno, "Detection of phosphatidylcholine oxidation products in rat heart using quadrupole time-of-flight mass spectrometry," Journal of Chromatography B, vol. 823, no. 1, pp. 37-43, 2005.

[14] F. M. Corrigan, D. F. Horrobin, E. R. Skinner, J. A. O. Besson, and M. B. Cooper, "Abnormal content of $n-6$ and $n-3$ longchain unsaturated fatty acids in the phosphoglycerides and cholesterol esters of parahippocampal cortex from Alzheimer's disease patients and its relationship to acetyl CoA content," International Journal of Biochemistry and Cell Biology, vol. 30, no. 2, pp. 197-207, 1998.

[15] Y. L. Ching, A. Lesimple, Å. Larsen, O. Mamer, and J. Genest, "ESI-MS quantitation of increased sphingomyelin in Niemann-Pick disease type B HDL," Journal of Lipid Research, vol. 46, no. 6, pp. 1213-1228, 2005.

[16] J. Silva, S. Dasgupta, G. Wang, K. Krishnamurthy, E. Ritter, and E. Bieberich, "Lipids isolated from bone induce the migration of human breast cancer cells," Journal of Lipid Research, vol. 47, no. 4, pp. 724-733, 2006.

[17] M. Okita, D. C. Gaudette, G. B. Mills, and B. J. Holub, "Elevated levels and altered fatty acid composition of plasma lysophosphatidylcholine(LysoPC) in ovarian cancer patients," International Journal of Cancer, vol. 71, no. 1, pp. 31-34, 1997.

[18] E. Iorio, D. Mezzanzanica, P. Alberti et al., "Alterations of choline phospholipid metabolism in ovarian tumor progression," Cancer Research, vol. 65, no. 20, pp. 9369-9376, 2005.

[19] P. Bougnoux, V. Chajes, M. Lanson et al., "Prognostic significance of tumor phosphatidylcholine stearic acid level in breast carcinoma," Breast Cancer Research and Treatment, vol. 20, no. 3, pp. 185-194, 1992.

[20] T. E. Merchant, J. N. Kasimos, T. Vroom et al., "Malignant breast tumor phospholipid profiles using ${ }^{31} \mathrm{P}$ magnetic resonance," Cancer Letters, vol. 176, no. 2, pp. 159-167, 2002.

[21] C. Wang, H. Kong, Y. Guan et al., "Plasma phospholipid metabolic profiling and biomarkers of type 2 diabetes mellitus based on high-performance liquid chromatography/electrospray mass spectrometry and multivariate statistical analysis," Analytical Chemistry, vol. 77, no. 13, pp. 41084116, 2005.

[22] Y. Wang, D. Botolin, J. Xu et al., "Regulation of hepatic fatty acid elongase and desaturase expression in diabetes and obesity," Journal of Lipid Research, vol. 47, no. 9, pp. 20282041, 2006.

[23] J.-C. Hogue, B. Lamarche, A. J. Tremblay, J. Bergeron, C. Gagné, and P. Couture, "Evidence of increased secretion of apolipoprotein B-48-containing lipoproteins in subjects with type 2 diabetes," Journal of Lipid Research, vol. 48, no. 6, pp. 1336-1342, 2007.

[24] S. J. Eun, Y. S. Hae, R. K. Mi et al., "Sphingosylphosphorylcholine induces proliferation of human adipose tissue-derived mesenchymal stem cells via activation of JNK," Journal of Lipid Research, vol. 47, no. 3, pp. 653-664, 2006.

[25] J. Laurencikiene, V. Van Harmelen, E. A. Nordström et al., "NF- $\kappa \mathrm{B}$ is important for TNF- $\alpha$-induced lipolysis in human adipocytes," Journal of Lipid Research, vol. 48, no. 5, pp. 10691077, 2007.

[26] P. Q. Tranchida, P. Donato, G. Dugo, L. Mondello, and P. Dugo, "Comprehensive chromatographic methods for the analysis of lipids," Trends in Analytical Chemistry, vol. 26, no. 3, pp. 191-205, 2007.

[27] S. Meier, S. A. Mjøs, H. Joensen, and O. Grahl-Nielsen, "Validation of a one-step extraction/methylation method for determination of fatty acids and cholesterol in marine tissues," Journal of Chromatography A, vol. 1104, no. 1-2, pp. 291-298, 2006. 
[28] M. P. Styczynski, J. F. Moxley, L. V. Tong, J. L. Walther, K. L. Jensen, and G. N. Stephanopoulos, "Systematic identification of conserved metabolites in GC/MS data for metabolomics and biomarker discovery," Analytical Chemistry, vol. 79, no. 3, pp. 966-973, 2007.

[29] J. C. Touchstone, "Thin-layer chromatographic procedures for lipid separation," Journal of Chromatography B, vol. 671, no. 12, pp. 169-195, 1995.

[30] J. K. Yao and G. M. Rastetter, "Microanalysis of complex tissue lipids by high-performance thin-layer chromatography," Analytical Biochemistry, vol. 150, no. 1, pp. 111-116, 1985.

[31] J. J. Myher and A. Kuksis, "General strategies in chromatographic analysis of lipids," Journal of Chromatography B, vol. 671, no. 1-2, pp. 3-33, 1995.

[32] C. Silversand and C. Haux, "Improved high-performance liquid chromatographic method for the separation and quantification of lipid classes: application to fish lipids," Journal of Chromatography B, vol. 703, no. 1-2, pp. 7-14, 1997.

[33] L. L. Dugan, P. Demediuk, C. E. Pendley II, and L. A. Horrocks, "Separation of phospholipids by high-performance liquid chromatography: all major classes, including ethanolamine and choline plasmalogens, and most minor classes, including lysophosphatidylethanolamine," Journal of Chromatography, vol. 378, no. 2, pp. 317-327, 1986.

[34] N. U. Olsson and N. Salem Jr., "Molecular species analysis of phospholipids," Journal of Chromatography B, vol. 692, no. 2, pp. 245-256, 1997.

[35] J. Becart, C. Chevalier, and J. P. Biesse, "Quantitative analysis of phospholipids by HPLC with light scattering evaporating detector-application to raw materials for cosmetic use," Journal of High Resolution Chromatography, vol. 13, pp. 126129, 1990.

[36] H.-Y. Kim, T.-C. L. Wang, and Y.-C. Ma, "Liquid chromatography/mass spectrometry of phospholipids using electrospray ionization," Analytical Chemistry, vol. 66, no. 22, pp. 39773993, 1994.

[37] P. J. Kalo, V. Ollilainen, J. M. Rocha, and F. X. Malcata, "Identification of molecular species of simple lipids by normal phase liquid chromatography-positive electrospray tandem mass spectrometry, and application of developed methods in comprehensive analysis of low erucic acid rapeseed oil lipids," International Journal of Mass Spectrometry, vol. 254, no. 1-2, pp. 106-121, 2006.

[38] B. Brügger, G. Erben, R. Sandhoff, F. T. Wieland, and W. D. Lehmann, "Quantitative analysis of biological membrane lipids at the low picomole level by nano-electrospray ionization tandem mass spectrometry," Proceedings of the National Academy of Sciences of the United States of America, vol. 94, no. 6, pp. 2339-2344, 1997.

[39] F. Süllentrop, D. Moka, S. Neubauer et al., "31 P NMR spectroscopy of blood plasma: determination and quantification of phospholipid classes in patients with renal cell carcinoma," NMR in Biomedicine, vol. 15, no. 1, pp. 60-68, 2002.

[40] J. M. Pearce and R. A. Komoroski, "Analysis of phospholipid molecular species in brain by ${ }^{31} \mathrm{P}$ NMR spectroscopy," Magnetic Resonance in Medicine, vol. 44, no. 2, pp. 215-223, 2000.

[41] J. Schille and K. Arnold, "Application of high resolution ${ }^{31} \mathrm{P}$ NMR spectroscopy to the characterization of the phospholipid composition of tissues and body fluids-a methodological review," Medical Science Monitor, vol. 8, no. 11, pp. 205-222, 2002.

[42] N. M. Loening, A. M. Chamberlin, A. G. Zepeda, R. G. Gonzalez, and L. L. Cheng, "Quantification of phosphocholine and glycerophosphocoline with ${ }^{31} \mathrm{P}$ edited ${ }^{1} \mathrm{H}$ NMR spectroscopy," NMR in Biomedicine, vol. 18, no. 7, pp. 413-420, 2005.

[43] J. Schiller, M. Müller, B. Fuchs, K. Arnold, and D. Huster,

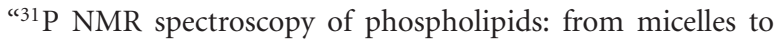
membranes," Current Analytical Chemistry, vol. 3, pp. 283301, 2007.

[44] D. Leibfritz, "An introduction to the potential of ${ }^{1} \mathrm{H}-,{ }^{31} \mathrm{P}-$ and ${ }^{13} \mathrm{C}$-NMR-spectroscopy," Anticancer Research, vol. 16, pp. 1317-1324, 1996.

[45] J. Henke, J. Engelmann, B. Kutscher et al., "Changes of intracellular calcium, fatty acids and phospholipids during Miltefosine-induced apoptosis monitored by fluorescenceand ${ }^{13}$ C NMR-spectroscopy," Anticancer Research, vol. 19, no. 5, pp. 4027-4032, 1999.

[46] J. Henke, W. Willker, J. Engelmann, and D. Leibfritz, "Combined extraction techniques of tumour cells and lipidphospholipid assignment by two dimensional NMR spectroscopy," Anticancer Research, vol. 16, no. 3, pp. 1417-1427, 1996.

[47] W. Willker and D. Leibfritz, "Assignment of mono- and polyunsaturated fatty acids in lipids of tissues and body fluids," Magnetic Resonance in Chemistry, vol. 36, no. 998, pp. S79S84, 1998.

[48] T. F. Bathen, J. Krane, T. Engan, K. S. Bjerve, and D. Axelson, "Quantification of plasma lipids and apolipoproteins by use of proton NMR spectroscopy, multivariate and neural network analysis," NMR in Biomedicine, vol. 13, no. 5, pp. 271-288, 2000.

[49] J. Willmann, K. Mahlstedt, D. Leibfritz, M. Spraul, and H. Thiele, "Characterization of sphingomyelins in lipid extracts using a HPLC-MS-offline-NMR method," Analytical Chemistry, vol. 79, no. 11, pp. 4188-4191, 2007. 

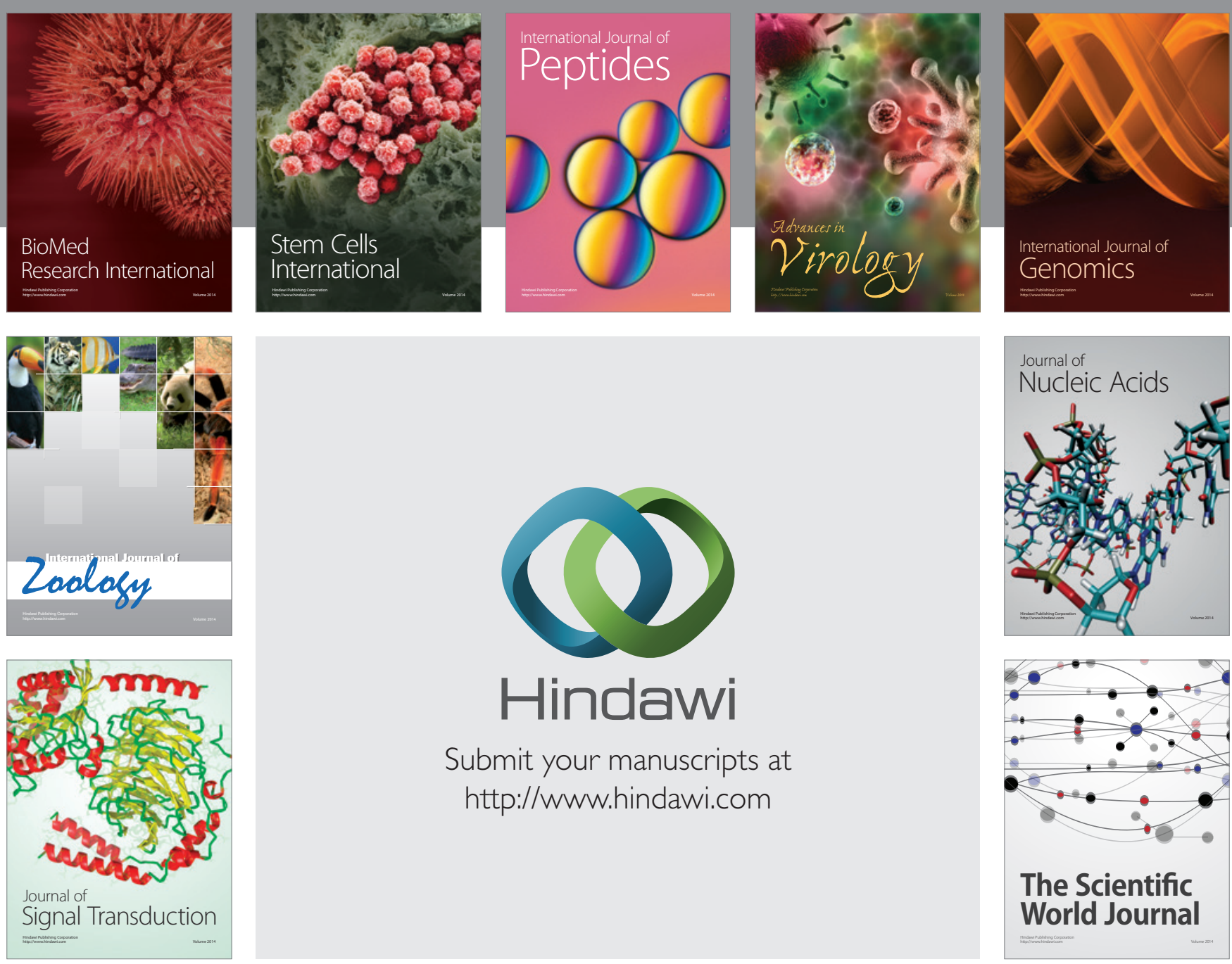

Submit your manuscripts at

http://www.hindawi.com
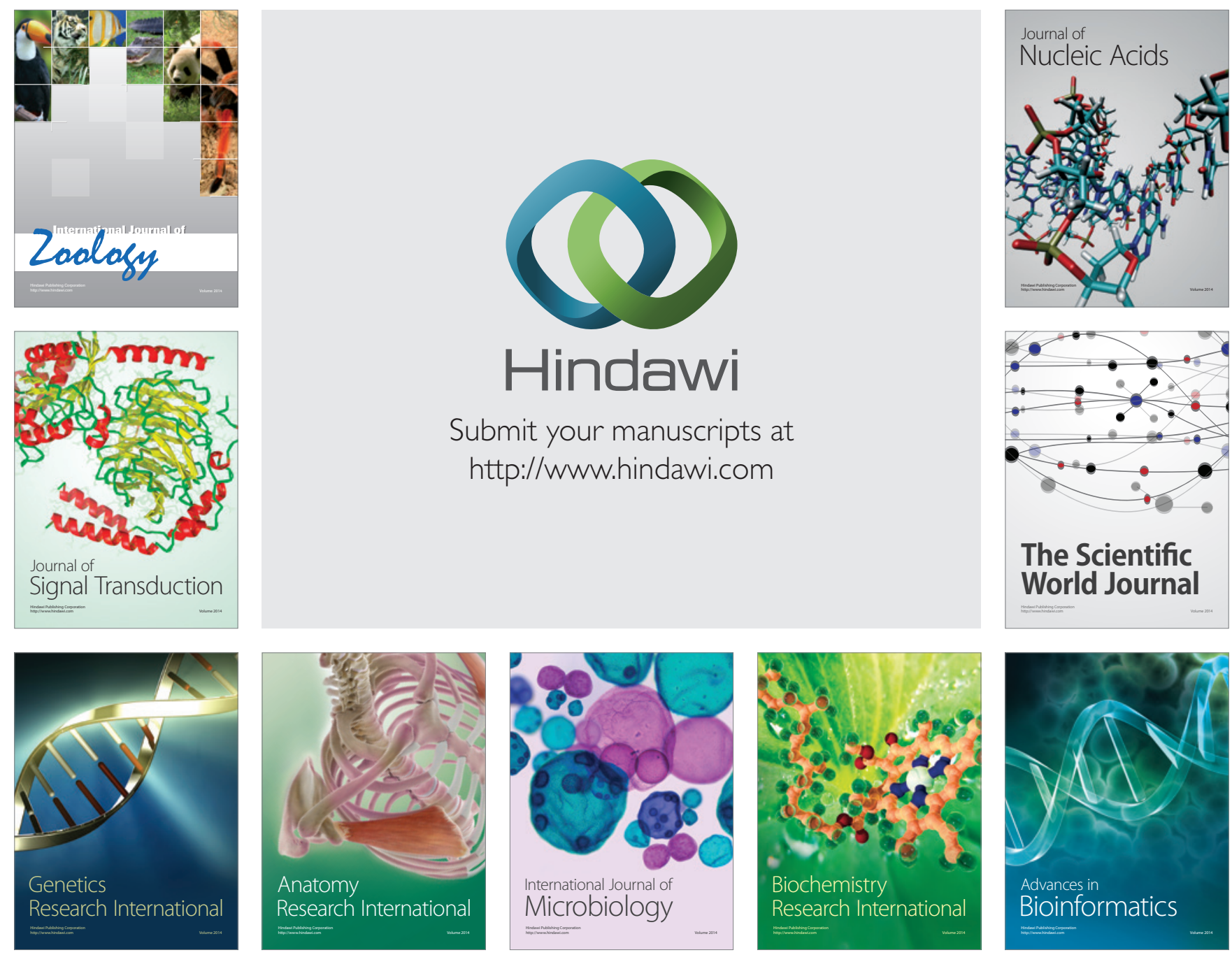

The Scientific World Journal
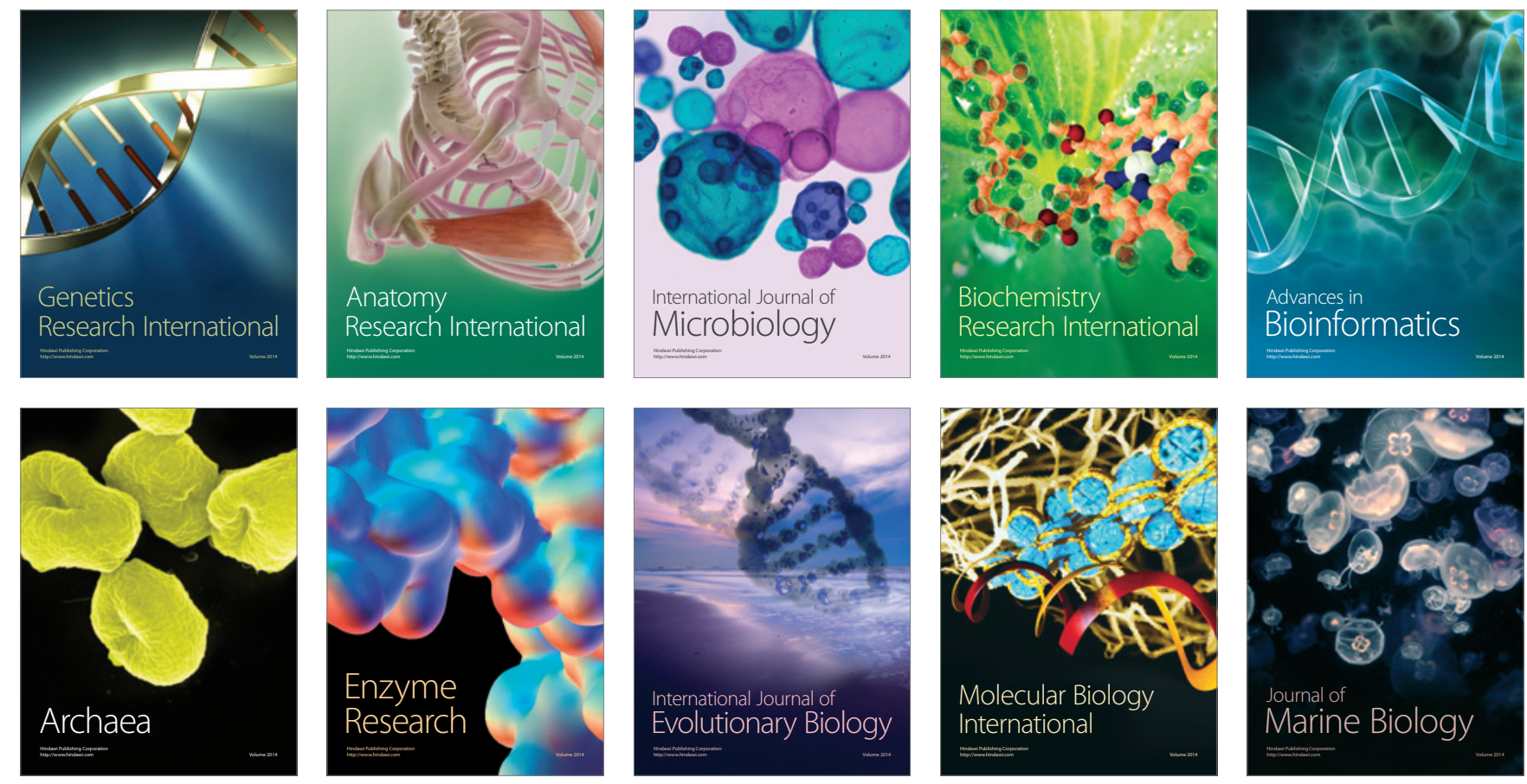